\title{
How to manage esophageal squamous cell carcinoma invading into the muscularis mucosa or submucosa after endoscopic resection
}

\author{
Hiroki Kurumi $^{1}$ (1) Takumi Onoyama $^{1} \cdot$ Hajime Isomoto $^{1}$
}

Received: 8 July 2021 / Accepted: 29 July 2021/Published online: 4 August 2021

(C) Japanese Society of Gastroenterology 2021

Keywords Esophageal squamous cell carcinoma . Endoscopic resection · Endoscopic submucosal resection · Additional treatment

The development and advancement of endoscopic resection (ER) techniques in recent years have enabled highly curative and minimally invasive treatment for esophageal squamous cell carcinoma (ESCC), with minimal risk of lymph node metastasis (LNM) [1]. With the aging of the population, there are high expectations for less invasive ER [2]. According to the latest Japanese guideline for ER of ESCC, cT1a-muscularis mucosa (MM)/cT1b-submucosa $\leq 200 \mu \mathrm{m}$ (SM1) ESCC is a relative indication for ER. Moreover, ER is the first therapeutic step in actual clinical practice [3, 4]. However, the management of pT1a$\mathrm{MM} / \mathrm{pT} 1 \mathrm{~b}-\mathrm{SM} 1 \mathrm{ESCC}$ following ER is challenging. This is because the current guideline does not provide definitive recommendations on the policy of additional treatment for pT1a-MM/pT1b-SM1 ESCC [3, 4]. The aforementioned guideline has not provided a recommendation statement for additional treatment in pT1a-MM ESCC with negative lymphovascular invasion (LVI). This can be attributed to the lack of reliable studies analyzing the benefit of additional treatment for pT1a-MM ESCC after ER. In contrast, additional treatment is recommended for patients with pT1b-SM ESCC, notwithstanding LVI or the depth of SM

Hiroki Kurumi

kurumi_1022_1107@yahoo.co.jp

1 Division of Gastroenterology and Nephrology, Department of Multidisciplinary Internal Medicine, Tottori University Faculty of Medicine, 36-1, Nishi-cho, Yonago,

Tottori 683-8504, Japan invasion. However, an appropriate treatment strategy for pT1b-SM1 with negative LVI is still controversial in real practice. This necessitates the treatment of pT1a-MM/ pT1b-SM1, despite limited evidence.

In the issue of "Journal of Gastroenterology", Hatta et al. conducted a multi-center retrospective study to investigate the metastatic recurrence rate and the prognostic impact of additional treatments in pT1a-MM/pT1bSM1 ESCC in patients who received ER. They classified pT1a-MM with negative LVI and vertical margin (VM), pT1b-SM1 with negative LVI and VM, and pT1b-SM2, positive LVI, or positive/unclear VM as categories A, B, and $\mathrm{C}$, respectively. The metastatic recurrence rate in categories A and B without additional treatment was $2.6 \%$ and $4.3 \%$, respectively. The metastatic recurrence rate was lower than those in previous reports that analyzed surgically resected specimens [5]. The tissue specimen slice width could affect LVI and depth of invasion assessment linked to proper assessment of LNM percentages with discordance between surgery and ERs in particular endoscopic submucosal dissection (ESD). The histopathological assessment is currently standardized using $2-3 \mathrm{~mm}$ thin slice specimens following en bloc ESD, thereby enabling accurate risk assessments of LVI. Furthermore, there was no significant difference in the metastatic recurrence rate and 5-year disease-specific survival (DSS) rate between categories $\mathrm{A}$ and $\mathrm{B}$, with and without additional treatment. Even in terms of overall survival (OS) rate, shown as supplemental information, pT1a-MM/pT1b-SM1 ESCC with negative LVI could be managed observationally with careful follow-up, presenting an alternative strategy of choice in elderly patients with less organ functions mostly due to comorbidities, given the invasiveness of additional 
current treatment which could induce critical adverse events.

Subsequently, the authors performed an analysis using their own new risk stratifications as follows: low-risk, pT1a-MM/pT1b-SM1 with negative LVI; intermediaterisk, pT1a-MM with LVI or pT1b-SM2; and high-risk, pT1b-SM with LVI. They observed significant DSS benefits in the low-risk group, compared to the latter groups. They could stratify metastasis risks predominantly belonging to LNM within the local region. The intermediate- and high-risk groups displayed a risk that was no less than 6 and 14 times over the low-risk group without additional treatment. Therefore, pT1b-SM1 without LVI cases could pose biological behavior similar to pT1a-MM negative for LVI in their larger cohorts. Prospective randomized controlled trials and case-control studies based on the results are awaited to confirm the aforementioned findings.

The authors also examined risk factors for metastatic recurrence, despite multi-center study design and the limitations. Lymphatic invasion, positive VM, and pT1b-SM2 were significant risk factors for metastatic recurrence. In category $\mathrm{C}$, the metastatic recurrence rates were significantly lower with additional treatment. Meanwhile, intermediate- and high-risk group affected DSS substantially and displayed stepwise metastatic risks without additional treatment. According to the study outcomes, we recommend additional treatment in cases of pT1a-MM/pT1bSM1 with LVI, pT1b-SM2, and positive VM following ER.

In conclusion, close follow-up with no additional treatment may be an acceptable option following ER in pT1aMM/pT1b-SM1 ESCC with negative LVI and VM. Meanwhile, the examination interval of 6-12 months appears considerably long than previous reports, such as JCOG 0508. Most metastatic events occurred within
12 months. Therefore, a closer follow-up with 3 months interval would be appropriate to identify metastatic loci linking to conduct timely additional treatment, considering the aggressive nature in certain ESCC subtypes. The OS was not affected by additional treatment. Moreover, elderly patients with ESCC have potential risks for the occurrence of the other organ malignancies and severe concomitant diseases. Thus, a careful follow-up is inevitable while conducting ER for cT1a-MM or cT1b-SM1 cases.

\section{Declarations}

Conflict of interest Author H.I is an Associate Editor of Journal of Gastroenterology. The remaining authors declare no conflict of interest for this article.

\section{References}

1. Abe S, Wu SYS, Ego M, et al. Efficacy of current traction techniques for endoscopic submucosal dissection. Gut Liver. 2020;14:673-84.

2. Hatta W, Gotoda $\mathrm{T}$, Koike $\mathrm{T}$, et al. Management following endoscopic resection in elderly patients with early-stage upper gastrointestinal neoplasia. Dig Endosc. 2020;32:861-73.

3. Ishihara R, Arima M, Iizuka T, et al. Endoscopic submucosal dissection/endoscopic mucosal resection guidelines for esophageal cancer. Dig Endosc. 2020;32:452-93.

4. Kitagawa Y, Uno T, Oyama T, et al. Esophageal cancer practice guidelines 2017 edited by the Japan Esophageal Society: part 1. Esophagus. 2019;16:1-24 (Springer Japan).

5. Eguchi T, Nakanishi Y, Shimoda T, et al. Histopathological criteria for additional treatment after endoscopic mucosal resection for esophageal cancer: Analysis of 464 surgically resected cases. Mod Pathol. 2006;19:475-80.

Publisher's Note Springer Nature remains neutral with regard to jurisdictional claims in published maps and institutional affiliations. 